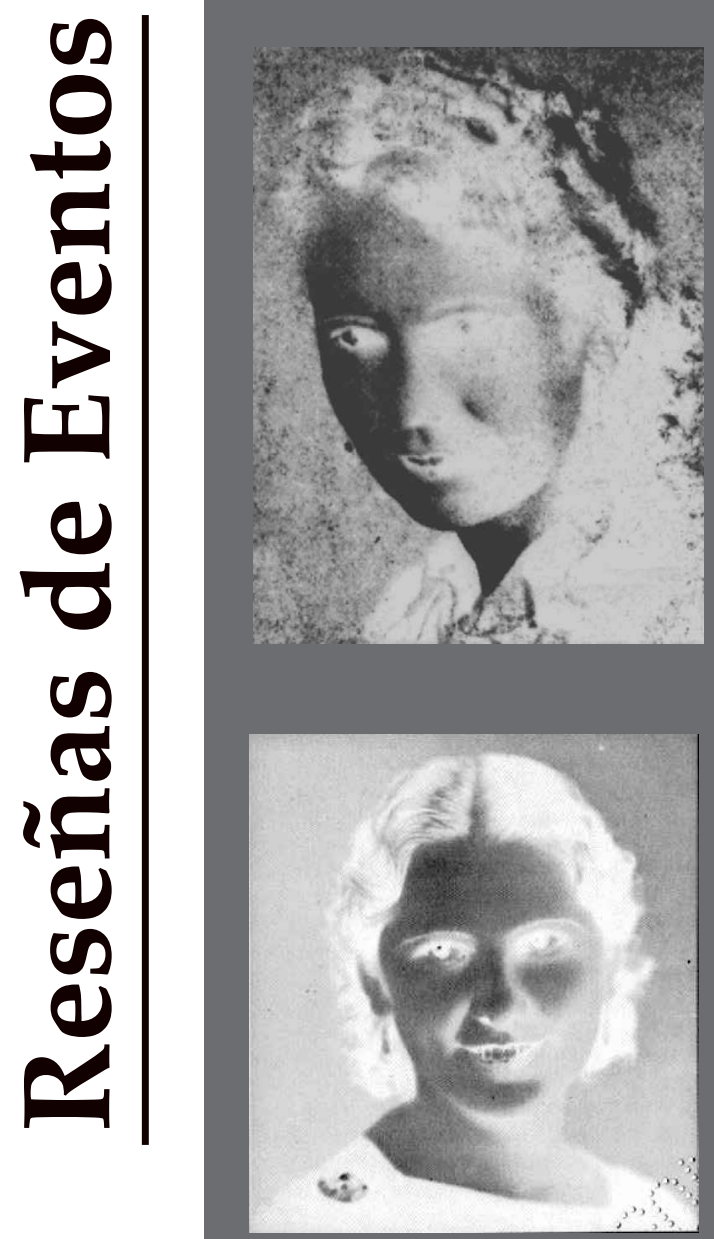

\title{
Eventos
}

\section{Reseñas Reseñas}

Reseñas

Reseñas de Eventos

Reseñas de Eventos

\section{Reseñas de Eventos}

Reseñas de Eventos

Reseñas de Eventos

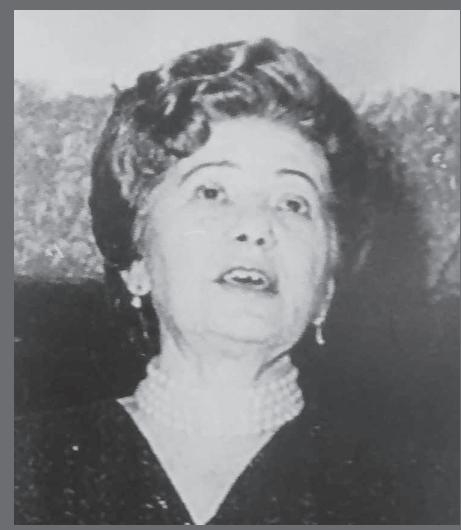




\section{PRESENTACIÓN DEL PROGRAMA NACIONAL DE BILINGUISMO 2015-2025}

Bogotá - Colombia, 10 de julio de 2013

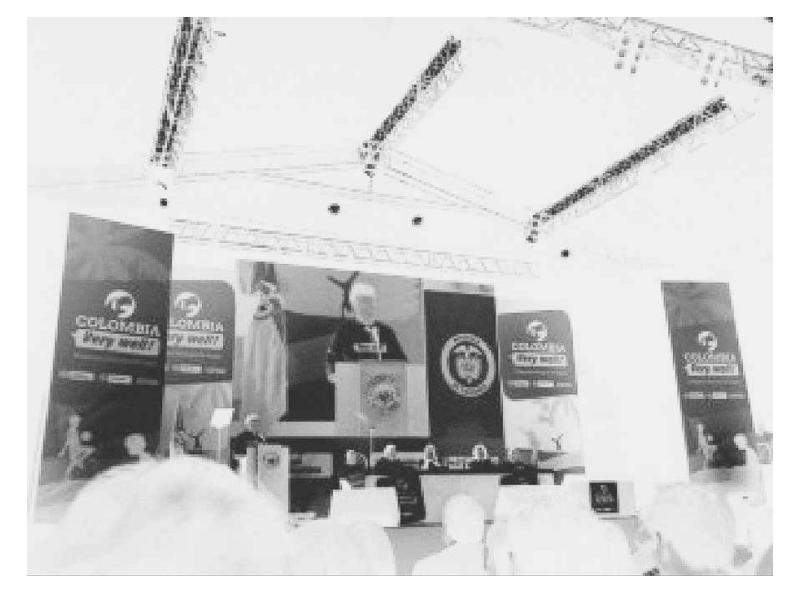

El citado evento presentó como objetivo el plantear, por parte del presidente de la República Juan Manuel Santos y de la Sra. Ministra de Educación María Fernanda Campo Saavedra, la política pública y el Plan Nacional de Bilinguismo 2015. Al acto asistieron los secretarios de Educación, gobernadores, rectores de las universidades, el presidente del Sistema Universitario Estatal Dr. Luis Enrique Arango y delegados del Consejo Nacional de Aseguramiento de la Calidad Superior (CONACES) y del Ministerio de Educación Nacional.

Se considera que "el manejo de una lengua extranjera es sin duda una habilidad que empodera a los individuos, les brinda mayores oportunidades de acceso al conocimiento y a otras culturas y los hace más competitivos". Desde esta perspectiva, el sector educativo se convierte en un factor relevante para consolidar una educación para el bilingüismo.

La señora ministra de Educación realizó un balance del proyecto de Fortalecimiento al Desarrollo de Competencias en Lenguas Extranjeras (20102014), donde manifestó que en el año 2013 el 43\% de los docentes de inglés que presentaron la prueba alcanzaron el nivel B2 frente al 29\% en el 2010, y entre los egresados de la educación, solo el 8\% lograron un nivel de B2. En la parte legal, el gobierno nacional ha reforzado con la Ley 1651 de 2013 y ahora inicia la implementación del programa que ha denominado Colombia Very Well, cuyo objetivo se enmarca en que todos los colombianos aprendan inglés. A este programa se tiene previsto invertir 1.3 billones de pesos para desarrollar los cinco componentes del citado programa Colombia Very Well. Por parte del Grupo 
de Investigación Historia y Prospectiva de la Universidad Latinoamericana (HISULA), asistieron al evento académico la Dra. Diana Soto Arango, de la Universidad Pedagógica y Tecnológica de Colombia, miembro de la Sala de Evaluación de Educación Consejo Nacional de Aseguramiento de la Calidad Superior (CONACES), y la profesora Liliana Paternina de la Universidad de Cundinamarca.

Liliana Paternina

Universidad de Cundinamarca

Grupo de investigación HISULA

\section{CONGRESO CENTROAMERICANO DE HISTORIA EN LA UNIVERSIDAD DE EL SALVADOR}

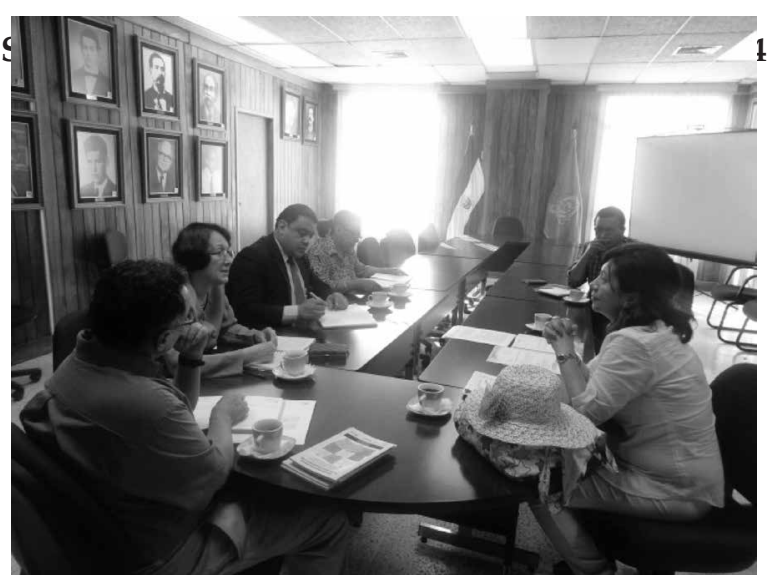

En el marco del XII Congreso Centroamericano de Historia desarrollado en la Universidad de El Salvador (UES), del 14 al 18 de julio del presente año, los grupos de investigación HISULA-ILAC se hicieron presentes con la comisión académica conformada por la Dra. Claudia Figueroa y el joven investigador Fredy Suárez. El congreso, celebrado en un país centroamericano cada dos años, contó con expertos investigadores a nivel local, nacional e internacional, los cuales presentaron excelentes conferencias magistrales, ponencias, talleres y exposiciones dirigidas al intercambio, debate y actualización de la comunidad de "centroamericanistas", desde el soporte académico e investigativo. Dentro de estas presentaciones, se destaca la conferencia magistral inaugural dada por el Dr. Víctor Hugo Acuña Ortega de la Universidad de Costa Rica, titulada: "Centroamérica en el mundo y el mundo en Centroamérica (siglos XIX y XX)", que resaltó la importancia que tiene Centroamérica, como punto geoestratégico, con relación al mundo entero, en el marco de giro globalizador que tuvo el 
mundo a partir de finales de la década del noventa, además de señalar el papel fundamental que tiene la historia en el análisis y comprensión de los procesos de globalización, entendiendo que "es la historia la única rama del conocimiento que nos dice qué fue el pasado, qué es el presente y cómo será el futuro".

En este evento participó la comisión de los grupos de investigación HISULA ILAC, representada por la Dra. Claudia Figueroa y el joven investigador Fredy Suárez, con la presentación de los resultados del proyecto de investigacion "Reformas, políticas públicas y movimientos universitarios" en el panel que lleva el mismo título y dentro de la sesión de la mesa temática "Rebeliones y movilización social, siglos XIX y XX", coordinada por los doctores Aarón Pollack y Ricardo Argueta. Estos son una muestra, a nivel internacional, de los procesos de investigación desarrollados en la universidad por los grupos de investigación HISULA-ILAC a lo largo de su existencia.

La labor de esta comisión académica fue la difusión de las redes de investigadores sobre las escuelas normales y reformas y movimientos universitarios y lograr la inscripción de algunos participantes al Congreso Centroamericano de Historia. Asimismo, se logró que esta comisión académica realizara contactos y compromisos académicos e investigativos con las directivas de la Universidad de El Salvador (UES), de gran interés para la comunidad académica de las dos universidades en las áreas de educación, ciencias sociales, medicina, derecho, entre otras.

El compromiso de los grupos de investigación HISULA-ILAC deja abiertos estos espacios de cooperación para que nuestra alma máter asuma los procesos a partir de la carta de intención enviada al señor rector de la UPTC de parte de las directivas de la Universidad de El Salvador. Las autoridades presentes en esta reunión fueron: la vicerrectora académica de la UES, MsD. Ana María Glower de Alvarado; el director del CIC-UES, Ing. Ramón Portillo; el secretario de Relaciones Internacionales, Mgs. Jorge Aranda; y el coordinador del INFORPUES, Lic. Carlos Alberto Ardón Gavarrete. En este sentido, es clara la intención de esta comunidad académica salvadoreña por realizar acercamientos para una posible firma de convenios entre los grupos de investigación de la UES, los grupos HISULA-ILAC y los programas de pregrado y posgrado de la UPTC. Finalmente, con el propósito de dar a conocer la producción académica de los grupos de investigación, se realizó la donación de los números 19, 20 y 21 de la Revista Historia de la Educación y de un ejemplar del libro La Escuela Rural en Colombia. Historias de Vida de Maestras. Mediados del siglo XX de la Dra. Diana Elvira Soto Arango a la Biblioteca Central de la UES. Nos llama la atención la admiración y el respeto por las universidades colombianas, específicamente por el trabajo que se está desarrollando desde la UPTC y los grupos de investigación 
con estos encuentros investigativos y académicos con miras a avanzar en la coordinación y cooperación institucional entre la UES y la UPTC, en un corto, mediano y largo plazo.

Fredy Giovany Suárez

Universidad Pedagógica y Tecnológica de Colombia Joven investigador (ILAC)

Claudia Figueroa

Universidad Pedagógica y Tecnológica de Colombia

( HISULA)

\title{
REFERENTES CULTURALES PARA LA PRIMERA INFANCIA AFRO, MINISTERIO DE CULTURA Y SU DIRECCIÓN DE POBLACIONES
}

\author{
Bogotá - Colombia, 28 de Julio de 2014
}

El Ministerio de Cultura y su Dirección de Poblaciones organizaron el día 28 de julio de 2014 el taller "Referentes culturales para la primera infancia afro", al cual asistieron académicos y miembros de organizaciones afros y representantes del Ministerio de Educación Nacional, Salud y Cultura.

El Grupo de Investigación Historia y Prospectiva de la Universidad Latinoamericana (HISULA), de la Universidad Pedagógica y Tecnológica de Colombia, presentó el proyecto de investigación "Maestras africanas y afrodescendientes", que lidera en convenio con: Grupo Rueca - Universidad de Cartagena, Grupo GIEM - Universidad Tecnológica del Chocó, Grupo Universidad de Nariño, Grupo EFPEM-USAC (Guatemala), Grupo UPROF UOP (Brasil), Grupo HEDURE -ULA (Venezuela), Grupo The Latin American - Caribbean Centre.The University of the West Indies (Jamaica), Grupo Hum 209-UPO (España), Grupo Docentes UNGE (Guinea Ecuatorial), Grupo Historia Latinoamericana - Universidad Omar Bongo (Gabón).

El citado proyecto presenta como objetivo "analizar y visibilizar el desempeño de las maestras africanas y afrodescendientes en lo socio-cultural, educativo, político, en la comunidad donde realizaron o están desarrollando la docencia con la incidencia en el entorno local y regional para establecer en qué medida las tradiciones culturales se mantienen vigentes en sus prácticas educativas y el 
liderazgo en la comunidad educativa" y se plantea la pregunta referentea: ¿inciden los imaginarios y convicciones de las maestras africanas y afrodescendientes, con su historia de vida, con el desarrollo de la práctica educativa e impacto sociocultural y político, en el cambio de mentalidades respecto a valores de solidaridad y tolerancia en Colombia, Guatemala, Venezuela, Jamaica, Gabón y Guinea Ecuatorial en los siglos XX y XXI?

El citado proyecto recibió comentarios favorables y se estableció el presentarlo para la respectiva financiación de la reunión prevista en febrero del 2015 en Guinea Ecuatorial. Se concluyó en la reunión la relevancia que la Universidad Pedagógica y Tecnológica de Colombia tiene en el contexto nacional al estudiar estos temas de poblaciones vulnerables y por tal motivo, junto con la universidad de Cartagena, sería llamada para liderar política pública referenciada al tema afro.

Es relevante conocer que en cuanto a la población de afros, negros, raizales y palenqueros, según el censo del DANE del año 2005, se reconocieron como afrocolombianos 4.311 .757 personas, lo que corresponde aproximadamente al 10,6\% del total de la población de Colombia.

Diana Elvira Soto Arango

Directora Proyecto de investigación

Directora grupo HISULA 


\section{INTERNACIONALIZACIÓN DE LOS PROYECTOS DE INVESTIGACIÓN DE LA UNIVERSIDAD PEDAGÓGICA Y TECNOLÓGICA DE COLOMBIA}

Kingston, Jamaica, 9 a 12 de septiembre de 2014

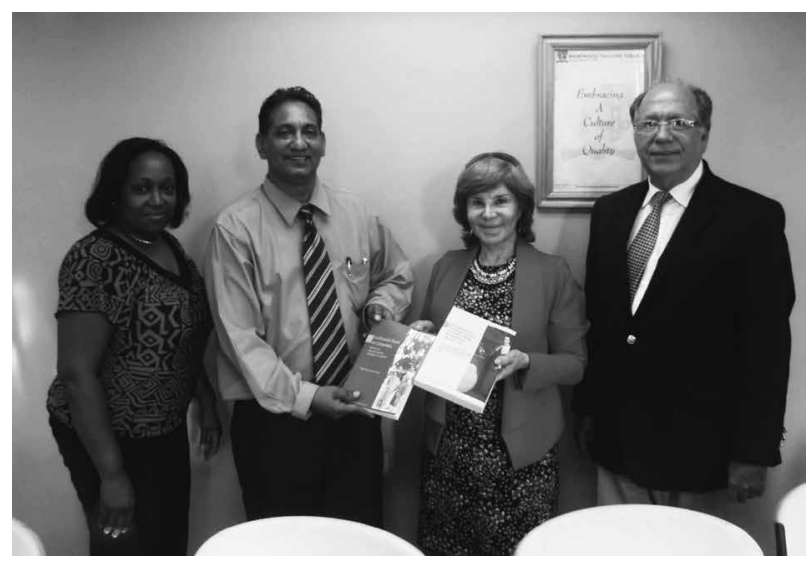

Foto 1: Entrega de Publicaciones Grupos HISULA-ILAC, docente del Teacher`s College - JamaicaFuente: Archivo Diana Elvira Soto.

Durante los días 9 a 12 de septiembre de 2014, en la ciudad de Kingston, Jamaica, la Dra. Diana Elvira Soto Arango, docente de la Universidad Pedagógica y Tecnológica de Colombia, socializó el proyecto de investigación de escuelas normales y avanzó en la organización de la propuesta de investigación "Maestras africanas y afrodescendientes" con instituciones universitarias y el Teacher's Colleges.

En esta corta estancia en la ciudad de Kingston la Dra. Soto logró la aprobación del Convenio Marco para RUDECOLOMBIA, movilidad para docentes y estudiantes de pregrado de la Universidad Pedagógica y Tecnológica de Colombia y, especialmente, organizó grupos de investigadores para la propuesta "Maestras africanas y afrodescendientes". Asimismo, se entrevistó con directivas e investigadores del Shortwood Teacher's College, de la University of the West Indies, instituciones escolares de primaria y las maestras que ingresarán al citado proyecto de investigación.

Por otra parte, se estableció con la embajada de Colombia en Jamaica la organización de un evento académico para celebrar los 200 años de la Carta de Jamaica con la coordinación del Grupo de investigación "La Ilustración en América Colonial" - ILAC_- 


\title{
XVIII JORNADA DE LA INVESTIGACIÓN: SIMPOSIO REFORMAS Y MOVIMIENTOS UNIVERSITARIOS GRUPOS DE INVESTIGACIÓN
}

\author{
Historia y prospectiva de la universidad latinoamericana. HISULA \\ La ilustración en América colonial. ILAC \\ Tunja, - Colombia, 7 y 8 Octubre del 2014
}

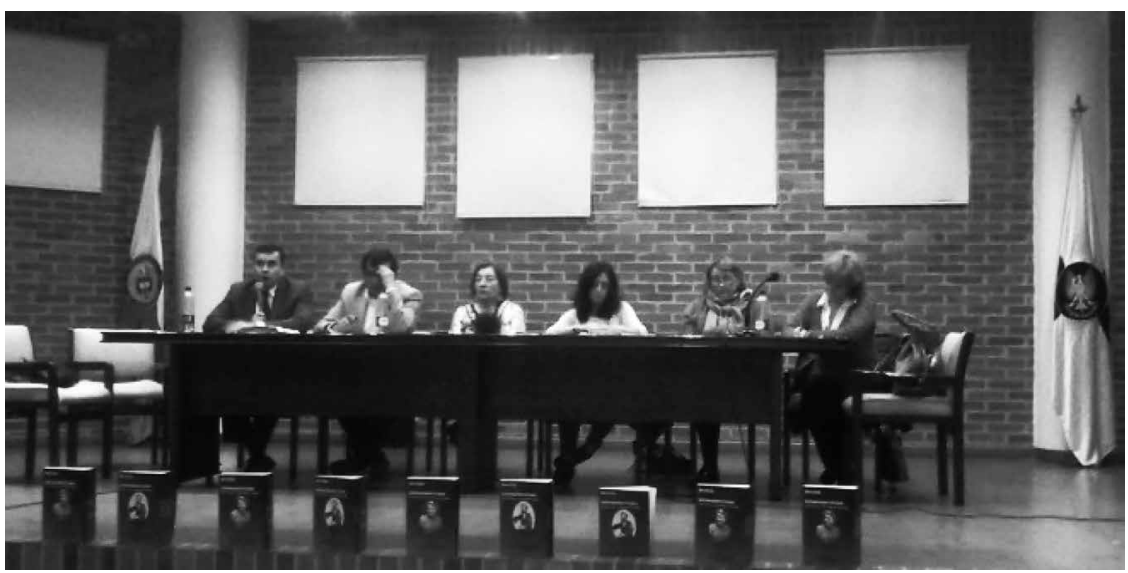

En el marco de la XVIII Jornada de la Investigación de la Universidad Pedagógica y Tecnológica de Colombia, los grupos de investigación HISULA - ILAC se hicieron partícipes con desarrollo del "Simposio, Reformas y Movimientos Universitarios", el cual se enmarca en el desarrollo del proyecto de investigación "Reformas, Políticas Públicas y Movimientos Universitarios" y de la Cátedra HISULA, en sus 11 años de existencia. El simposio se desarrolló durante los días 7 y 8 de octubre del 2014 en el Auditorio Rafael Azula de la UPTC, contando de manera permanente con un promedio de 150 asistentes de profesores y estudiantes de pregrado y posgrado de la universidad. El simposio fue coordinado por la Dra. Diana Elvira Soto Arango, directora de los grupos de investigación. El simposio se realizó en tres paneles y un conversatorio, de la siguiente manera;

Panel 1: La participación del movimiento universitario y su incidencia en la construcción de la política educativa en Iberoamérica.

Panel 2: La participación del movimiento universitario y su incidencia en la construcción de la política educativa en Colombia (1970-2014).

Conversatorio: Prospectiva de la Universidad del siglo XXI.

Panel 3: Estado actual y dinámica del movimiento universitario colombiano.

En el primer panel se contó con la participación de las invitadas internacionales Dra. Renate Marsiske de la UNAM (México), Dra. Remedios Ferrero Micó 
de la Universidad de Valencia (España) y la Mgs. Olga Pérez Molina de la Universidad San Carlos de Guatemala (Guatemala). En el segundo, con los invitados nacionales Dr. Álvaro Acevedo Tarazona (UIS) y Dr. Carlos Alberto Molina (UPTC) y la presentación de la ponencia de la Dra. Elizabeth Vidal Arizabaleta (CESU), realizada por la Dra. Diana Elvira Soto. Para el desarrollo del conversatorio se contó con la participación de nuestros invitados nacionales e internacionales y con las intervenciones por parte del auditorio. Finalmente, el tercer panel contó con la participación de los estudiantes Carlos Mario Restrepo y Jairo Andrés Rivera, miembros de la MANE y de la Asociación Colombiana de Estudiantes Universitarios (ACEU) y de la Federación de Estudiantes Universitarios (FEU), respectivamente.

El evento se desarrolló por la convocatoria interna de apoyo a eventos realizada por la Dirección de Investigación (DIN) de la Universidad Pedagógica y Tecnológica de Colombia. Entre las conclusiones podemos destacar que el movimiento universitario ha presentado una invisibilidad histórica de sus protagonistas y que la construcción de la universidad es incierta porque aún no se sabe bajo cuáles parámetros se va a evolucionar; de ahí la gran responsabilidad de los actuales estudiantes universitarios en la construcción colectiva de esta universidad del futuro. Queda por señalar que el desarrollo de este importante evento representa un aporte más por parte de los grupos HISULA-ILAC, bajo el aval de Sociedad Historia de la Educación Latinoamericana (SHELA), en la construcción de una universidad guiada por la discusión académica, la reflexión colectiva y el pensamiento crítico que desde la Universidad Pedagógica y Tecnológica de Colombia nos proyectamos hacia Iberoamérica.

Fredy Giovany Suárez Guacaneme Universidad Pedagógica y Tecnológica de Colombia Joven investigador (ILAC) 


\section{$X$ CONGRESO DE LA SOCIEDAD DE HISTORIA DE LA EDUCACIÓN LATINOAMERICANA.}

\section{Puerto Vallarta - México - 26 de octubre al 02 de Noviembre de 2014}

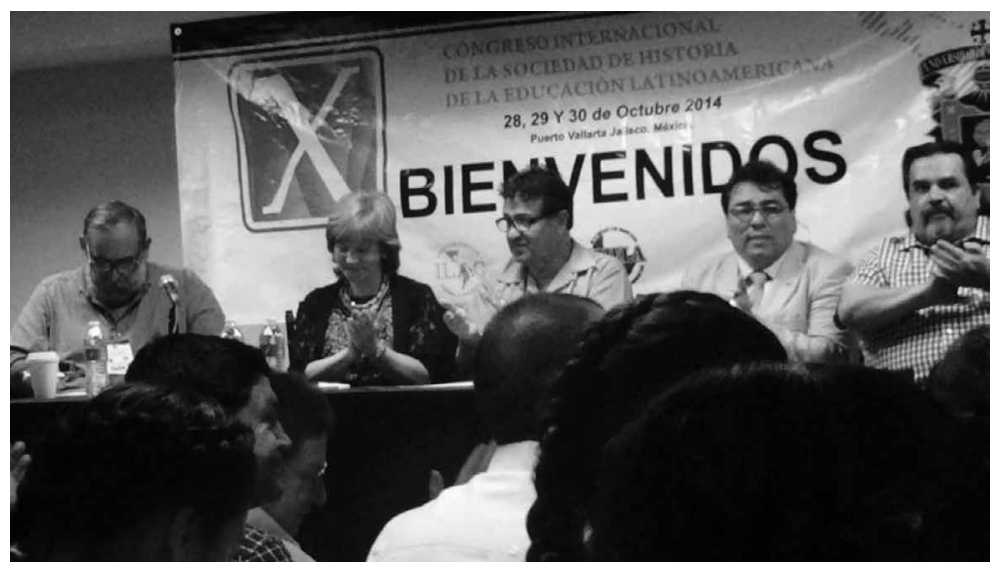

El X congreso de la Sociedad de Historia de la Educación Latinoamericana es un certamen académico que se realiza cada dos años, bajo la coordinación de la mesa directiva y el comité científico de la SHELA; en esta oportunidad el congreso se realizó en la ciudad de Pto. Vallarta del estado Jalisco, México, bajo la colaboración de la Universidad de Guadalajara y con la excelente coordinación del Dr. Armando Martínez Moya (investigador de HISULA) y del equipo de trabajo de los grupos de investigación Historia y Prospectiva de la Universidad Latinoamericana (HISULA) y La Ilustración en América Colonial (ILAC). La actividad de participación en el X congreso inicia con la propuesta de presentar cuatro simposios bajo los temas que se adelantan en los proyectos de investigación vigentes. En este sentido, desde Colombia se adelantó la formulación de los simposios, así como su divulgación y convocatoria, y por ende la recepción de trabajos de ponencia. En esta actividad se recibieron más de 97 ponencias de 12 países (Guatemala, Argentina, Perú, Ecuador, Honduras, México, Venezuela, Cuba, Brasil, Estados Unidos de Norteamérica, Canadá, España y Colombia). Estos trabajos se remitieron a la coordinación general, que presentó los simposios como mesas de trabajo: Mesa 1. Políticas públicas, reformas y movimientos estudiantiles y magisteriales. Festividades, publicaciones, vidas, recuerdos, imágenes; Mesa 7. Simposio: Prensa ilustrada en América Latina; Mesa 5. Historia de la formación de maestros/as y de las escuelas normales en Iberoamérica; y la Mesa 6. Simposio: Maestras africanas y afrodescendientes en Colombia, Brasil, Guatemala, Venezuela, Guinea Ecuatorial Siglo XX a XXI.

En cada uno de los espacios de construcción y reflexión se presentaron, desde distintas perspectivas y contextos, investigaciones que permitieron adelantar un 
balance del estado actual de los estudios en el campo de la historia social de la educación en América Latina, para finalmente descubrir que existen momentos y coyunturas que coinciden en el tiempo histórico de cada uno de los países que se vieron representados a través a de sus investigadores(as). Asimismo, se compartieron experiencias de investigación y se adelantaron planes de trabajo desde la proyección de los proyectos en curso.

La historia de la educación en América Latina comparte momentos determinantes para su historia tanto en el terreno de la formación de educadores y escuelas normales como en el tema de las reformas educativas y los movimientos universitarios. Una de las explicaciones que van a servir de reflexión es el hecho de que las políticas educativas en cada momento de la historia responden a coyunturas geopolíticas. La educación ha sido el vehículo por el cual se ha reproducido la ideología de los gobiernos y, en la misma vía, ha sido asumida como el motor que moviliza la economía de una nación. Desde estas dos perspectivas, y en el marco de las tensiones y antagonismos político-ideológicos acaecidos con más fuerza en el siglo XX, se ubica la educación, en la cual se han desarrollado las ideas educativas con perspectiva latinoamericana a través de los movimientos magisteriales y universitarios. Por otro lado, resulta relevante indicar que la Dra. Diana Elvira Soto presentó el proyecto de investigación que avanza sobre la perspectiva de las historias de vida, en este caso de maestras africanas y afrodescendientes, desde el análisis comparado. Con este propósito trabajó en más de 12 países de América Latina y el Caribe, Guinea y Gabón y avanzó a través de grupos de investigación en la recolección de la información, así como dando a conocer la propuesta con el fin de vincular, a través de la firma de protocolos de actividades, a grupos de investigación e instituciones educativas y gubernamentales interesadas en el proyecto.

Por otro lado, se debe hacer mención a los reconocimientos otorgados este año por la SHELA, que distinguió con el Botón de Oro al Dr. Luis Enrique Arango, exrector de la Universidad Tecnológica de Pereira. Asimismo, se hizo entrega del Premio Joven Investigador “Diana Elvira Soto Arango" al joven investigador Diego Eduardo Naranjo por la propuesta investigativa presentada. También cabe mencionar que se inauguró el reconocimiento a la actividad editorial, otorgado a la magíster Sandra Bernal, docente de tiempo completo de la Escuela de Ciencias Sociales UPTC y asistente editorial de RHELA.

Asimismo, desde la mesa directiva del congreso se aprobó un pronunciamiento en rechazo al caso de los 43 normalistas de Ayotzinapa desaparecidos, en el que se exige se esclarezcan los hechos y se llama a la solidaridad de la comunidad académica para con los familiares de los estudiantes desaparecidos, así como para con los normalistas mexicanos. 
En todo caso, la posibilidad brindada por este importante encuentro académico es precisamente la de compartir estas reflexiones, pensar en colectivo los problemas de la educación en América Latina desde una perspectiva histórica y, sin duda, adelantar la tarea de construir y avanzar en propuestas por alcanzar un sistema educativo incluyente que desde la reflexión histórica avance en la consecución de una educación humanizante, que además propenda por una verdadera integración latinoamericana.

Diego Eduardo Naranjo Joven investigador (ILAC)

\section{PRE- POST Y DESARROLLO DEL VIII SEMINARIO TALLER CENTRO INVESTIGACIÓN INTERNACIONAL. CENTRO DE INVESTIGACIÓN INTERNACIONAL EN HUMANIDADES Y EDUCACIÓN. VENDIMIA,}

Tunja - Colombia, 2 al 9 de noviembre de 2014

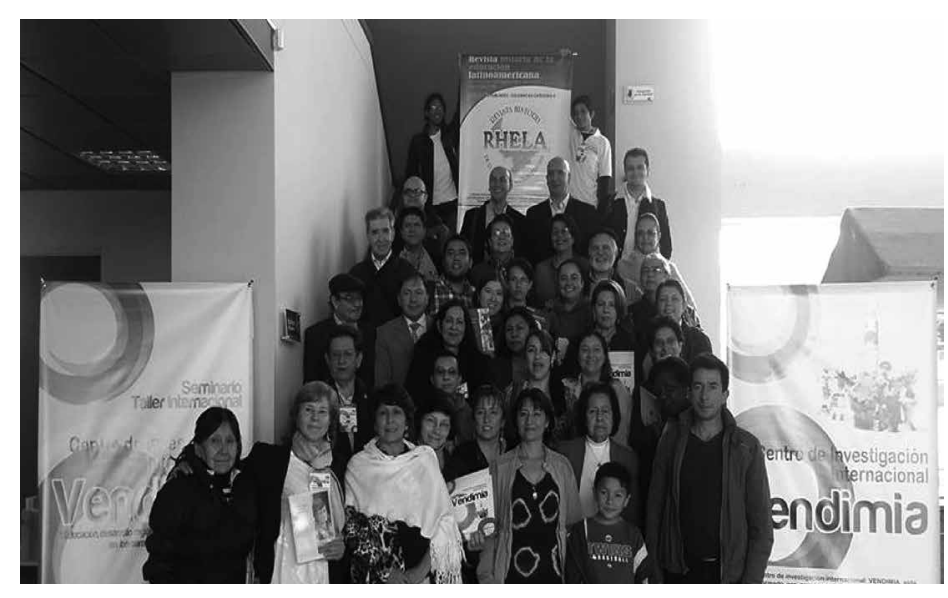

Las actividades realizadas del 2 al 9 de noviembre del 2014 comprenden el pre, el post y el desarrollo del VIII Centro Investigación Internacional VENDIMIA.

En primera instancia, se destacan las actividades para la organización del VIII Seminario Taller Centro Investigación Internacional VENDIMIA. En nuestro caso, con los grupos de investigación Historia y prospectiva de la Universidad Latinoamericana (HISULA) y La Ilustración en América Colonial (ILAC), se organizaron cuatro simposios desde los proyectos de investigación que se tienen inscritos en la DIN y los nuevos que se están organizando y de los cuales ya se tienen avances. En dichos simposios se presentaron 45 ponencias, 
de las cuales 18 correspondían a investigadores internacionales procedentes de siete países. La organización de los citados cuatro simposios se estableció desde la programación que realizan los grupos desde octubre de cada año para la realización en el siguiente año. Asimismo, en esta organización colaboramos con grupos de menor desarrollo de la Universidad Pedagógica y Tecnológica de Colombia con el objetivo de que se vayan desarrollando en la investigación y en la visibilidad de sus resultados. Por lo anterior, con un equipo de docentes y jóvenes investigadores se realizaron los cronogramas de actividades a los 18 investigadores internacionales y 27 nacionales que están vinculados en alguno de nuestros proyectos de investigación y pertenecen a los grupos, redes y protocolos que se han firmado con sus instituciones. Por este motivo, los pasajes en el $70 \%$ se pagaron por la universidad de procedencia. Los siete países de origen de los investigadores internacionales que tienen relación con los proyectos de investigación de HISSULA-ILAC son: Brasil, Costa Rica, Canadá, España, Guatemala, México y Venezuela. Se debe destacar que estos investigadores internacionales y varios nacionales participaron no solo como ponentes en el Centro de Investigación Internacional VENDIMIA, sino que además realizaron actividades de asesoría de tesis, jurado de tesis (4), cursos en el doctorado, seminario taller dirección de tesis doctorales para los docentes del doctorado y graduados, conferencias especiales para el pregrado, paneles y asesoría a trabajos a jóvenes investigadores.

Respecto a la primera actividad del pre-VENDIMIA, se realizó el Seminario Taller de Dirección de Tesis, que se coordinó entre la Dra. Diana Elvira Soto Arango y el Dr. Carlos Londoño. A esta actividad asistieron representantes de Guatemala, Brasil, Canadá, España y profesores del doctorado en Ciencias de la Educación, y se concluyó con recomendaciones tomando en cuenta las experiencias de los países participantes. Se realizó el día 4 de noviembre. En segundo lugar se encuentra el Foro de Candidatos a la Rectoría, que se organizó con los jóvenes investigadores desde el proyecto de investigación "Políticas públicas, reformas y movimientos universitarios" y la cátedra de Historia de la universidad. El foro se realizó en el Teatro Fausto de la Universidad Pedagógica y Tecnológica de Colombia, con una asistencia promedio de 300 personas y con la participación de seis candidatos de los ocho que se presentan a la rectoría. Fue el único acto académico que realizó una actividad con una amplia consulta estudiantil, profesoral y de investigadores sobre la universidad latinoamericana. Se recibieron mil preguntas de estudiantes, 200 de profesores, 50 de administrativos y 100 de investigadores internacionales. Las preguntas se clasificaron en 11 temas. Esta clasificación se realizó en conjunto con los coordinadores del foro: Dr. Justo Cuño, Dra. Elizabeth Vidal, Dra. Diana Elvira Soto Arango y el secretario del foro el Lic. Fredy Suárez, joven investigador de La Ilustración en América Colonial (ILAC). 
La tercera actividad se desarrolló el día miércoles 5 de noviembre con un panel sobre "Pedagogías. Modelos en los Doctorados Ciencias de la Educación". Lo coordinó el Dr. Wilson Valenzuela y participaron académicos de España, Brasil, Guatemala, Venezuela y Canadá. También hicieron parte 65 personas entre profesores y estudiantes de doctorado y de pregrado. Al finalizar el panel se firmaron los protocolos de actividades entre el Grupo de Investigación Historia y Prospectiva de la Universidad Latinoamericana (HISULA) y otros grupos de investigación para el proyecto de investigación "Maestras africanas y afrodescendientes". Igualmente, se presentó el Tomo VII de la colección Educadores que se editó en la Universidad San Carlos de Guatemala sobre el tema de maestras rurales en Guatemala.

La cuarta actividad se concretó en el seminario de tesis a los estudiantes del doctorado en Ciencias de la Educación, y nos acompañaron profesores de México, España y Guatemala. Durante el evento del Centro de Investigación Internacional VENDIMIA, coordinado por la Dra. Alba Nidia Triana del 6 al 8 de noviembre de 2014, se organizó, desde los grupos de investigación Historia y Prospectiva de la Universidad Latinoamericana (HISULA) y La Ilustración en América Colonial (ILAC), la coordinación de cuatro simposios de los 10 que se llevaron a cabo en el seminario taller. Todos los simposios obedecieron a los proyectos de investigación que estamos realizando en los citados grupos de investigación. En los simposios se presentaron 45 ponencias de las 116 que se sustentaron en el Centro de Investigación Internacional VENDIMIA. En estas ponencias participaron 18 investigadores internacionales. Destacamos por su entidad que la Dra. Teresa Sierra de la Universidad Pedagógica Nacional de México socializó en este evento los resultados de la investigación del postdoctorado que realizó en la Universidad Pedagógica y Tecnológica de Colombia (Acuerdo 064 del 31 de octubre de 2008) en el Grupo de Investigación Historia y Prospectiva de la Universidad Latinoamericana (HISULA), bajo la dirección de la Dra. Diana Elvira Soto Arango. Igualmente, inició su actividad postdoctoral en el doctorado en Ciencias de la Educación (CADE) de la Universidad Pedagógica y Tecnológica de Colombia la Dra. Josefina Quintero de la Universidad de Caldas, Colombia, y se encuentra en desarrollo del posdoctorado el Dr. Álvaro Acevedo de la UIS de Santander.

Asimismo se participó con los invitados internacionales en actividades del doctorado en Ciencias de la Educación en calidad de jurados en exámenes de suficiencia y en sustentaciones de tesis. Por otra parte, se realizaron dos reuniones de la junta directiva del Centro de Investigación Internacional VENDIMIA.

En el post-VENDIMIA se realizó el III Seminario Taller de Autoevaluación del CADE de la UPTC, con la participación de 25 personas entre estudiantes, 
profesores, egresados y administrativos. Lo relevante es que se revisó y se dieron recomendaciones al reglamento estudiantil del doctorado en Ciencias de la Educación de RUDECOLOMBIA. Para el equipo de jóvenes investigadores de los grupos de investigación HISULA-ILAC, Mónica, Ángela, Diego y Fredy, los asistentes Diana María Bastidas y Yaneth Vargas, la coordinadora de la cátedra "Historia y Prospectiva de la Universidad Latinoamericana" (HISULA) profesora Sandra Bernal, Dra. Celina Trimiño, Dra. Alba Nidia Triana y la candidata a doctor Yaneth Ayala, nuestro agradecimiento por el apoyo en la organización de estas actividades y a los investigadores extranjeros, que una vez más han demostrado su apoyo y compromiso con las actividades académicas del doctorado en Ciencias de la Educación y a los grupos de investigación HISULA- ILAC. Asimismo, a la Dra. Elizabeth Vidal y Justo Cuño por el trabajo exhaustivo para llevar a cabo el "Foro de Candidatos a la Rectoría de la Universidad Pedagógica y Tecnológica de Colombia 2015-2018”.

Diana Elvira Soto Arango

Directora grupos de investigación HISULA-ILAC

PRE-SEMINARIO TALLER VENDIMIA-2014

"FORO DE CANDIDATOS A LA RECTORÍA DE LA UPTC 2015 - 2018"

CATEDRA HISTORIA Y PROSPECTIVA DE LA UNIVERSIDAD

LATINOAMERICANA

PROYECTO DE INVESTIGACIÓN “REFORMAS, POLÍTICAS

PÚBLICAS Y MOVIMIENTOS UNIVERSITARIOS,

Tunja - Colombia, 4 de noviembre de 2014

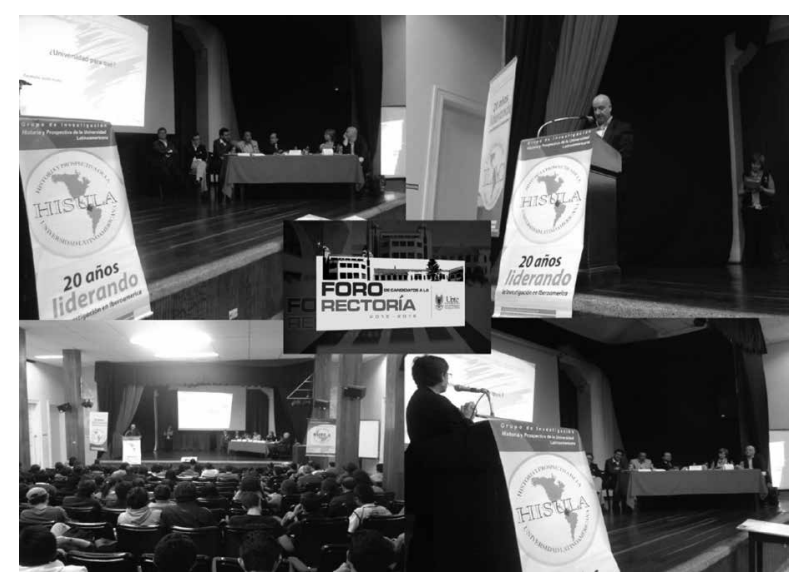


Ante la importancia que marca el desarrollo de los actuales comicios electorales para la elección del rector de la Universidad Pedagógica y Tecnológica de Colombia (UPTC), cabe destacar que se merece un profundo compromiso, desde la academia, por construir nuevos escenarios para el diálogo, la discusión y la construcción colectiva de una propuesta de universidad, acorde a las más sentidas necesidades de la comunidad académica UPTCista.

Por tal razón, los grupos de investigación Historia y Prospectiva de la Universidad Latinoamericana (HISULA) (Categoría A) y La Ilustración en América Colonial (ILAC) (Categoría A1), coordinados por la Dra. Diana Elvira Soto Arango, se dieron a la tarea de desarrollar el "Foro de Candidatos a la Rectoría de la UPTC 2015-2018", el martes 4 de noviembre de 2014, en las instalaciones de la universidad. Contó con la participación de seis de los ocho candidatos inscritos y avalados por el Consejo Superior y con una nutrida asistencia entre estudiantes de pregrado y posgrado, profesores y administrativos.

En este sentido, el Foro de Candidatos se desarrolló a partir de la convocatoria a cada uno de los candidatos a la rectoría y de la debida invitación a los diferentes estamentos que componen la universidad para contar con su participación en este escenario abierto al diálogo académico y argumentado.

Con miras a dar mayor dinámica y organización al evento, los grupos HISULA e ILAC abrieron sus redes sociales, correos y demás plataformas virtuales para la recepción de más de mil preguntas de estudiantes, 200 de profesores, 50 de administrativos y 100 de investigadores internacionales dirigidas a los candidatos y formuladas por los diferentes estamentos de la universidad. Destacamos por la relevancia las 100 preguntas de la comunidad académica internacional, en el marco del X Congreso de la Sociedad de Historia de la Educación Latinoamericana realizado en Puerto Vallarta, México, del 27 al 31 de octubre de 2014. La sistematización, categorización y definición de los núcleos temáticos y preguntas que serían realizadas a los candidatos se llevó a cabo por parte de los coordinadores del foro y el secretario de éste. En la realización se contó con todo el apoyo de los jóvenes investigadores de los grupos HISULA-ILAC, la coordinadora de la cátedra HISULA y profesores de la Escuela de Sociales y del doctorado en Ciencias de la Educación. Se destaca que en la moderación del foro se contó con la participación y colaboración del docente e investigador de la Universidad Pablo de Olavide (España) Dr. Justo Cuño Bonito, de la Dr. Elizabeth Vidal Arizabaleta, miembro del Consejo Nacional de Educación Superior (CESU), y de la Dra. Diana Soto, quienes desarrollaron el foro durante tres horas bajo las 11 preguntas que correspondían a 11 temáticas clasificadas por las preguntas allegadas. El foro contó en promedio con la asistencia de más de 300 personas. 
En definitiva, el desarrollo del "Foro de Candidatos a la Rectoría de la UPTC 2015-2018" representó una apuesta por parte de los grupos de investigación HISULA-ILAC en el avance y desarrollo de una investigación para la democracia universitaria y comprometida profundamente con la potenciación de la academia. También se debe destacar que dicho escenario fue reconocido y apoyado por todos los estamentos de la universidad, por otras universidades públicas del país, por el propio Consejo Nacional de Educación Superior y por la comunidad académica internacional y demás cuerpos e instituciones que conforman el sistema universitario de Colombia, convirtiéndose en una experiencia importantísima en la construcción de una academia científica, investigativa y democrática.

Finalmente, nos queda por señalar que la realización del foro se enmarca en el desarrollo del Proyecto de Investigación "Reformas, Políticas Públicas y Movimientos Universitarios", avalado por Colciencias y que lleva a cabo el grupo de investigación ILAC, además de articularse con la cátedra HISULA en sus 11 años de existencia, como propuesta de extensión académica y científica liderada por el grupo de investigación HISULA.

Agradecemos a todos el respaldo, acompañamiento y participación en el desarrollo del Foro de Candidatos. A la Escuela de Ciencias Sociales (UPTC), a la Facultad de Ciencias de la Educación (UPTC), al doctorado en Ciencias de la Educación (RUDECOLOMBIA-UPTC), a la Universidad Pablo de Olavide, al Consejo Nacional de Educación Superior (CESU), a la Sociedad de Historia de la Educación Latinoamericana (SHELA) y en general a la comunidad académica nacional e internacional.

Fredy Giovany Suárez Joven Investigador - ILAC

\title{
PANEL: PEDAGOGÍA, TENDENCIAS ACTUALES DE LOS DOCTORADOS EN EDUCACIÓN DE COLOMBIA
}

\author{
Tunja - Colombia, 4 de noviembre de 2014
}

El panel "Pedagogía, tendencias actuales de los doctorados en educación de Colombia" fue organizado por los grupos de investigación Historia y Prospectiva de la Universidad Latinoamericana (HISULA) y La Ilustración en América Colonial (ILAC) el día 5 de noviembre, como parte de las actividades académicas previstas para el inicio del VIII Seminario Taller Internacional Vendimia 2014. El evento contó con la asistencia de estudiantes y docentes del doctorado en Ciencias de la Educación y de la Escuela de Ciencias Sociales mayoritariamente. 
El panel se llevó a cabo en el auditorio $N^{\circ} 1$ del Edificio Administrativo de la UPTC, fue coordinado por el Dr. Wilson Valenzuela, y la secretaría fue realizada por las jóvenes investigadoras del grupo Hisula Ángela Fonseca y Mónica Valbuena. Además, contó con la participación de tres ponentes: el Dr. Oscar López de la Universidad San Carlos de Guatemala, la Dra. Théresse Hamel de la Universidad Aval (Quebec- Canadá) y la Dra. Violeta Núñez Pérez de la Universidad de Barcelona (España); y tres contraponentes: Dr. José Rubens Lima Jardilino de la Universidad de Ouro Preto (Brasil), Dra. Josefina Quintero de la Universidad de Caldas y el Dr. Pascual Mora de la Universidad de Los AndesTáchira (Venezuela).

Durante el desarrollo del panel los ponentes, a través de sus experiencias educativas e investigativas, socializaron sus conclusiones frente a las vicisitudes y encrucijadas por las cuales atraviesa la educación superior, que debe responder a las demandas de un mercado global sin dejar de lado las fronteras del currículo y las necesidades del contexto local. Por lo tanto, se planteó la necesidad de crear un currículo que tenga como prioridad los procesos de aprendizaje individual y colectivo desde una pedagogía de la comprensión, que permita crear y mantener culturas propias de las territorialidades como las representaciones y formas de identidad de los pueblos nativos, negros, raciales.

Ángela Fonseca

Mónica Valbuena

Jóvenes Investigadoras -HISULA-

\title{
ACTIVIDADES VIII SEMINARIO TALLER INTERNACIONAL. VENDIMIA \\ VIII SEMINARIO TALLER INTERNACIONAL VENDIMIA RESEÑAS DE SIMPOSIOS
}

\author{
Tunja - Colombia. 6 al 8 noviembre de 2014 \\ MESA 1. ESCUELAS NORMALES E INSTITUCIONES FORMADORAS DE \\ MAESTROS EN AMÉRICA LATINA EN EL MARCO DEL VIII SEMINARIO TALLER \\ Centro de Investigación Internacional en Humanidades y Educación. VENDIMIA,
}

La mesa No. 1, titulada Escuelas normales e instituciones formadoras de maestros en América Latina, fue coordinada por los investigadores: Claudia Figueroa (Colombia), Justo Cuño Bonito (España) y José Rubens Lima Jardilino (Brasil). En el simposio participaron 15 ponentes provenientes de los países de Brasil, Colombia, Guatemala y México. 
Se enmarca dentro del proyecto de investigación "Maestras y escuelas normales en Colombia durante los siglos XIX y XX", que se coordina entre el grupo de investigación Historia y Prospectiva de la Universidad Latinoamericana (HISULA) (Categoría A Colciencias), financiado por la Universidad Pedagógica y Tecnológica de Colombia, y el grupo Formación de profesorado, de la Universidad San Carlos de Guatemala. El simposio presentó como objetivo analizar la trayectoria histórica de las escuelas normales en América Latina como la institución que forma maestros y maestras, partiendo del estudio desde Hispanoamérica, ya que conforme a como se van constituyendo las repúblicas en sus respectivas leyes se consigna la posibilidad de abrir instituciones que formen maestros para que los Estados puedan desarrollar una política educativa coherente con los anhelos e ideales de sus líderes.

Se concluyó que en la mayoría de los países estas instituciones solo trascendieron con un mayor énfasis a partir de la década de los años setenta del siglo XIX. Su consolidación a través de políticas educativas permitió su auge durante el siglo $\mathrm{XX}$, inmerso en un proceso fragmentado de modernización en muchos de los países de América Latina.

Pese a las dificultades de financiación y a los irregulares resultados en las distintas naciones latinoamericanas, los estudios normalistas se irán consolidando con planes de estudio aprobados a nivel nacional pero que en la práctica fueron adecuados a las características específicas de su entorno socioeconómico. En ese entorno, las maestras y los maestros normalistas formaron a las nuevas generaciones de los estudiantes latinoamericanos en los valores tradicionales emanados de sus entornos locales, regionales y nacionales, acordes a una normativa estatal, en teoría, pero en la práctica íntimamente vinculados a las costumbres, creencias, representaciones y usos de los contextos sociales y culturales más inmediatos.

Mónica Lizet Valbuena Porras Joven investigadora -HISULA

\section{RESEÑA MESA $\mathrm{N}^{\circ} 2$ \\ MAESTRAS AFRICANAS Y AFRODESCENDIENTES EN COLOMBIA, BRASIL, GUATEMALA, VENEZUELA, GUINEA ECUATORIAL SIGLO XX A XXI}

En el marco del VIII Seminario Taller Internacional VENDIMIA se socializaron los avances investigativos del proyecto marco titulado "Maestras africanas y afrodescendientes en Colombia, Brasil, Guatemala, Venezuela, Guinea Ecuatorial siglo XX a XXI", propuesto por el grupo de investigación Historia y Prospectiva de la Universidad Latinoamericana y adelantado conjuntamente con destacados grupos de investigación nacionales e internacionales. 
Las experiencias formativas e investigativas se centralizaron en la mesa de trabajo $\mathrm{N}^{\mathrm{o}} 2$, cuyo nombre coincide con el mencionado proyecto, desarrollada los días 6 y 7 de noviembre, coordinada por el Dr. José Cortez, Pascual Mora y la Dra. Diana Soto, y con la secretaría de Ángela Fonseca, joven investigadora del grupo HISULA. La mesa fue inaugurada con el panel titulado "Encuentros y desencuentros teóricos sobre los estudios afroamericanos", en el que participaron el Dr. José Cortez (Guatemala), Pascual Mora (Venezuela), Luz Marina (Colombia), Rafael Díaz Díaz (Colombia), y que fue coordinado por la Dra. Diana Elvira Soto Arango y la Dra. Gabriela Hernández. De igual modo, se socializaron cinco ponencias, que destacaron el papel protagónico de algunas maestras afrodescendientes en las trasformaciones educativas y sociales de su región a través de su historia de vida.

\section{Ángela Fonseca Joven investigadora -HISULA- \\ RESEÑA MESA $\mathrm{N}^{\circ} 3$ REFORMAS Y MOVIMIENTOS UNIVERSITARIOS}

La mesa de trabajo $\mathrm{N}^{\circ}$ 3, "Reformas y Movimientos Universitarios", coordinada por el Dr. Álvaro Acevedo Tarazona y la Dra. Diana Elvira Soto Arango, con la secretaría de los grupos de investigación HISULAILAC, a través del joven investigador Fredy Suárez, tuvo lugar el 6 y 7 de noviembre en el Auditorio $\mathrm{N}^{\circ} 1$ del Edificio Administrativo de la UPTC. Esta mesa se desarrolla en el marco del proyecto de investigación "Reformas, Políticas Públicas y Movimientos Universitarios", realizado por el grupo de investigación La Ilustración en América Colonial (ILAC).

La mesa contó con un panel inaugural de apertura sobre reformas universitarias en España, México, Guatemala y Venezuela, y con la participación de los investigadores internacionales Miguel Ángel Beas (España), ArmandoMartínez Moya(México), DaniloLópez(Guatemala)y José Pascual Mora (Venezuela), así como de 13 ponentes entre Colombia, España y México, que desarrollaron temáticas sobre las reformas universitarias, las transformaciones de la universidad, la mujer en la educación y la historia de los movimientos universitarios en Iberoamérica. El desarrollo de esta mesa muestra como conclusión la importancia y avance del estudio de la universidad y la educación, al tiempo que evidencia el creciente interés por ahondar en el estudio de los movimientos universitarios y su relación con la universidad, la educación y la sociedad. Se debe indicar que en este simposio se incluyó el simposio sobre prensa ilustrada en América Latina que coordinó el grupo La Ilustración en América Colonial (ILAC), desde el proyecto de investigación "La prensa ilustrada en América Latina y el 
Caribe", sobre el cual se viene avanzando en convenio con la Universidad de Barranquilla, a través del Dr. Jairo Solano Alonso.

Fredy Suárez

Diego Eduardo Naranjo

Jóvenes Investigadores -HISULA-ILAC-

\section{RESEÑA SUB-MESA. PRENSA ILUSTRADA EN AMÉRICA LATINA}

El simposio se realizó el día 7 de noviembre de 2014 en el marco del VIII Seminario Taller Internacional VENDIMIA, dentro de la Mesa 3: "Reformas y Movimientos Universitarios". Esta submesa estuvo coordinada por el Dr. Jairo Solano Alonso y contó con la secretaría del joven investigador Diego Naranjo y con la colaboración del joven investigador Fredy Suárez. La convocatoria a este simposio de especialistas fue atendida por tres expertos en esta línea de investigación, dos ponencias por Colombia, una sobre el origen de la prensa en el Caribe colombiano, y otra sobre la discusión en torno a la reproducción ideológica que se da a través de los textos escolares en América Latina; por otro lado, el Dr. Justo Cuño Bonito, de la Universidad Pablo de Olavide (España), presentó una interesante ponencia, que sin duda sintetiza y da claridad sobre una perspectiva en investigación que parte de estudiar, a través de la vida de un personaje (biografía), los hechos y acontecimientos históricos de un período histórico, permitiendo la reconstrucción del contexto analizado.

Este simposio se realizó en el marco del desarrollo del proyecto de investigación "La prensa ilustrada en América Latina y el Caribe", el cual se viene formulando desde el pasado IX Coloquio de Historia Social y de las Ciencias realizado entre el 20 y el 22 de marzo de 2014 en la ciudad de Cartagena, que bajo el tema de "La masonería en la independencia americana" introdujo el tema de la prensa como medio a través del cual se difundió el pensamiento ilustrado en América Latina y el Caribe. En este sentido, se propuso como conclusión este tema de investigación que el grupo de investigación ILAC asumió con el fin de presentarlo formalmente dentro del plan de acción del año 2015.

Diego Eduardo Naranjo Joven Investigador -ILAC-

CONCLUSIONES, III Taller Auto-evaluación CADE-UPTC, 8 noviembre 2014

Primera Parte. Conferencia: Proceso de autoevaluación con fines de acreditación de alta calidad en un programa de doctorado en red, bajo los lineamientos del CNA, por la Dra. Diana Lago, directora de acreditación institucional en la Universidad de Cartagena. 
1. El programa de doctorado en Ciencias de la Educación es un solo programa, el cual se direcciona desde una sola unidad curricular. Se pueden hacer modificaciones consultando al Ministerio de Educación.

2. Puntos básicos: Para la renovación se realizó un documento por cada CADE y uno general. El programa funciona bajo el principio de la corresponsabilidad: el programa no es de nadie pero es de todos, y hay que ofrecerlo en una unidad académico-administrativa. El registro lo tiene la Universidad Pedagógica y Tecnológica de Colombia, y todo el trámite se debe realizar desde esta institución.

3. La autoevaluación se concibe como un autoexamen de un solo proceso. Los programas en red se legitiman a través de los convenios, los cuales se deben cumplir. El doctorado es un solo programa; no son diez. La normativa es la de la red. La doctora Diana Lago manifestó que se refiere al convenio específico académico-administrativo que le da el funcionamiento de red al programa por el cual se dio el registro calificado.

4. Este proceso de revisión del reglamento estudiantil es para el proceso de autoevaluación con fines de hacer procesos de mejora. El punto de partida es el modelo de autoevaluación, el cual fue aprobado por concejo de rectores y se presentó con el documento maestro. Es el referente que tiene el Ministerio de Educación; si queremos autoevaluarnos con un modelo diferente, hay que legitimarlo y enviarlo al Ministerio de Educación. Este modelo tiene una metodología, dada por el CNA.

5. No es conveniente terminar con la articulación porque aparece en el documento maestro. La articulación tiene grandes beneficios para los estudiantes. Cuando se inicia un proceso de autoevaluación, hay que conocer la historia para mejorarla. La articulación de las maestrías con los doctorados es una tendencia mundial. En el ejercicio de autoevaluación que se ejecutó desde los CADE en los años 2009 a 2010 se encontró como debilidad el factor profesores porque no tienen dedicación de tiempo completo, mientras que en la investigación se deben incrementar políticas y estrategias en cada universidad: por ejemplo, darle más fuerza a VENDIMIA y fortaleza a la internacionalización, que se ha convertido en el factor no solo para el programa sino que debe ser transversal para los demás. Hay que tener un diagnóstico del doctorado en Ciencias de la Educación y diseño de planes de mejoramiento. Asimismo, es importante que los planes de mejoramiento se evalúen constantemente porque es lo que más se tiene en cuenta en la autoevaluación. Se debe cumplir los reglamentos: por ejemplo, para ser director del CADE se requiere 
ser director de un grupo A o B; para ser director de tesis, se debe estar habilitado; para ser miembro del CADE y docente, se deben cumplir unos requisitos. El punto clave es la internacionalización: todo el mundo sabe que se está exigiendo el inglés.

6. En el programa del doctorado en Ciencias de la Educación los factores y las características deben estar acorde con la misión y visión del programa. Lo que se está pidiendo como autoevaluación son los planes de mejoramiento, que deben ser de corto y de mediano plazo. La evaluación debe ser permanente; y no se debe dejar de lado la ponderación, que es un ejercicio colectivo.

7. En la Universidad de Cartagena se hizo un proyecto de comunicación iniciado por la doctora Rina de León con los estudiantes de comunicación social y trabajo social. La ponderación es netamente académica y corresponde directamente a cada CADE. Primero se ponderan las características de cada factor y, posteriormente, los factores. Este es un ejercicio cualitativo al que por razones prácticas se le da un valor numérico. En el doctorado de Cartagena cada asistente tiene un proyecto de mejora dirigido por la doctora Diana Lago; las asistentes trabajan por proyectos, no por tareas. El proceso de autoevaluación se convirtió en una escuela de pensamiento.

8. En definitiva, Punto uno: partir de que es un programa en red en el que lo más importante es su colectivo. Punto dos: el modelo en Cartagena ya se actualizó al 2013; este es un ejercicio interno pero no vale para RUDECOLOMBIA, solo para Cartagena. Punto tres: hay que anotar que el MEN exige dos documentos de autoevaluación y dos evaluaciones de planes de mejora, que puede pedir en cualquier momento. Punto cuatro: la ponderación es un ejercicio del mayor rigor; tiene que ser participativa y NO consiste en un ejercicio matemático. La ponderación es un ejercicio cualitativo, y su gran importancia está en que define el perfil del programa. Punto cinco: en el proceso de recolección de información es importante definir cuál herramienta tecnológica se va a utilizar; ésta debe ser la misma para todas las universidades de la red. Por último: se hace el análisis de resultados a nivel general y particular, y lo mismo para los planes de mejora; el modelo tiene dos aspectos: apreciación y evaluación.

9. Finalmente, recuerda que en este momento las universidades deben tener mínimo un $40 \%$ de los programas de posgrados acreditados si desean reacreditarse institucionalmente. 


\section{Taller de "Análisis Nuevo Reglamento Estudiantil" \\ Doctorado Ciencias de la Educación. RUDECOLOMBIA}

La doctora Diana Soto explica el procedimiento que se utilizó para analizar el reglamento estudiantil teniendo en cuenta el acuerdo 002 del 2008, el documento que elaboró el Comité Curricular que se tomó como base, y los acuerdos 052 y 025 de la UPTC de 2012.

Para el inicio del análisis se presentaron las recomendaciones que sobre este aspecto se tenían en la tesis de Mario Torres. Seguidamente, la estudiante Mary Luz Báez indicó los aspectos jurídicos que se debían tener en cuenta. Se estableció una comisión de cinco estudiantes para la validez de lo acordado ante la ausencia del representante de los estudiantes. Asimismo, se preguntó por la no asistencia del director encargado del CADE y se indicó por parte de varios estudiantes que el director les había indicado que no era importante asistir a este taller de autoevaluación. La Dra. Soto informó que tanto el estudiante como el director (E) habían manifestado que tenían asuntos personales que atender. En el taller se trabajó de manera colectiva; la estudiante Mercedes Cárdenas realizó la secretaría, y se analizó cada artículo dando sugerencias en cada caso. Se anexa el documento con las recomendaciones sugeridas.

En general, se encuentra en los reglamentos de RUDECOLOMBIA y en el de la Universidad Pedagógica y Tecnológica de Colombia, de postgrados que es necesario reglamentar y visibilizar 1 a internacionalización del currículo que la entendemos, en primera instancia, como la carta de navegación sustentada en la formación de valores interculturales con capacidad de adaptar e integrar en crítica constructiva y de manera creativa otras culturas al entorno local desde la sociedad del conocimiento ${ }^{1}$. Segundo, se concibe desde la interdisciplinariedad y la flexibilidad que permita las homologaciones en una movilidad transversal de los estudiantes donde el bilingüismo es un requisito básico en el dialogo de saberes entre las instituciones para las dobles titulaciones. Tercero, es el conjunto de estrategias evidenciadas en las líneas de investigación, proyectos(con firma de protocolos de actividades entre grupos de investigación), en el plan de estudios con el dominio y aplicación de las tics, el bilingüismo (en las lecturas, ponencias, seminarios), movilidad transversal de los docentes nacionales e internacionales a través de redes de investigación que conlleven a la calidad académica del programa haciendo del mismo un punto de referencia para los investigadores y estudiantes internacionales. En definitiva, el programa debe articular nuevos procesos sociales, que

1 "El conocimiento se ha convertido en objeto de inmensos desafíos económicos, políticos y culturales, hasta tal punto que las sociedades cuyos contornos empezamos a vislumbrar bien pueden calificarse de sociedades del conocimiento. BINDE, Jerónimo (2005): Hacia las sociedades del conocimiento. Informe mundial de La UNESCO. París, Ediciones UNESCO. Publicado en 2005 por la Organización de las Naciones Unidas, para la Educación, la Ciencia y la Cultura.7 Place de Fontenoy; 75732 París 07 SP. www. unesco.org/publications (consultada el 25 de mayo del 2010), p. 5. 
involucran espacios físicos, mentales, de comunicación y nuevos actores internacionales ${ }^{2}$.

Diana Elvira Soto Arango

Coordinadora Auto-evaluación

CADE-UPTC

2 Soto Arango Diana (2011):"La Universidad Latinoamericana. Un futuro en construcción”, en Independencia e Universidade NaAmérica Latina. Tradicoes, Tempos e territórios. Sao Paulo.Paco Editorial. Sociedad de Historia de la Educación Latinoamericana, pp. 193-234. Soto Arango Diana, Uribe Jorge Tomás, Acuña, Olga y otros (2009).La construcción de la nación Iberoamericana. Siglos XIX a XXI.T unja, en Cuadernos de VENDIMIA I y II, T unja, RUDECOLOMBIA, COLCIENCIAS, CADE Universidad Pedagógica y Tecnológica de Colombia, HISULA. 